CARDIOVASCULAR MEDICINE

\title{
Cardiovascular, cerebrovascular, and respiratory changes induced by different types of music in musicians and non-musicians: the importance of silence
}

\author{
L Bernardi, C Porta, P Sleight
}

See end of article for authors' affiliations .....................

Correspondence to: Dr Luciano Bernardi, Clinica Medica 2 Universita' di Pavia, IRCCS S Matteo, 27100 Pavia, Italy;lbern1ps@unipv.it

Accepted 18 August 2005 Published Online First 30 September 2005

\begin{abstract}
Objective: To assess the potential clinical use, particularly in modulating stress, of changes in the cardiovascular and respiratory systems induced by music, specifically tempo, rhythm, melodic structure, pause, individual preference, habituation, order effect of presentation, and previous musical training. Design: Measurement of cardiovascular and respiratory variables while patients listened to music. Setting: University research laboratory for the study of cardiorespiratory autonomic function. Patients: 12 practising musicians and 12 age matched controls.

Interventions: After a five minute baseline, presentation in random order of six different music styles (first for a two minute, then for a four minute track), with a randomly inserted two minute pause, in either sequence.

Main outcome measures: Breathing rate, ventilation, carbon dioxide, RR interval, blood pressure, midcerebral artery flow velocity, and baroreflex.

Results: Ventilation, blood pressure, and heart rate increased and mid-cerebral artery flow velocity and baroreflex decreased with faster tempi and simpler rhythmic structures compared with baseline. No habituation effect was seen. The pause reduced heart rate, blood pressure, and minute ventilation, even below baseline. An order effect independent of style was evident for mid-cerebral artery flow velocity, indicating a progressive reduction with exposure to music, independent of style. Musicians had greater respiratory sensitivity to the music tempo than did non-musicians.

Conclusions: Music induces an arousal effect, predominantly related to the tempo. Slow or meditative music can induce a relaxing effect; relaxation is particularly evident during a pause. Music, especially in trained subjects, may first concentrate attention during faster rhythms, then induce relaxation during pauses or slower rhythms.
\end{abstract}

M usic now has an increasing role in several disparate areas. Music can reduce stress and improve athletic performance, motor function in neurologically impaired patients with stroke or parkinsonism, or milk production in cattle. ${ }^{1-10}$

Listening to music is a complex phenomenon, involving psychological, emotional, neurological, and cardiovascular changes, with behavioural modifications of breathing. ${ }^{11}{ }^{12}$ Non-musicians listen by using the non-dominant hemisphere, whereas musicians (who are probably more attentive) use the dominant hemisphere. ${ }^{13}$ These responses may be influenced by musical style (for example, classical versus rock), melody, harmonic structure, rhythm, and tempo but also by verbal content-for example, the brain asymmetry shown for language and melody perception has not been found in rhythm perception. ${ }^{13}$ Heart rate, blood pressure, or respiration have been studied. ${ }^{1}{ }^{14} 15$ So far, however, autonomic, cardiovascular, and respiratory changes in response to such a large range of music, order of presentation, or the effect of a short interpolated pause, and the responses related to musical training have not been comprehensively compared. Cardiorespiratory variables can be modified by rhythmic repetition of a prayer or a yoga mantra or by recitation of poetry. ${ }^{16}{ }^{17}$ We therefore investigated whether listening to music has similar effects.

We investigated the responses to six types of music (with differing rhythmic, harmonic, and melodic structures) in musicians versus non-musicians. We measured cardiovascular, respiratory, and cerebrovascular variables and their short term reproducibility and habituation, as well as the non-specific effect of a random order of presentation.

\section{METHODS}

Subjects

The protocol was approved by the local ethics committee; all subjects gave informed consent. We studied 24 healthy right handed white subjects (mainly colleagues or medical students; table 1). Twelve had no previous special training in music (non-musician group; table 1), whereas 12 were advanced conservatoire students or post-conservatoire diplomates, with at least seven years of continuous practice (musician group; table 1). The main instruments played were violin (four), piano (three), flute (two), clarinet (one), trumpet (one), and bass (one). Some had experience with other modern instruments (electric guitar (two) and drums (one)).

\section{Study protocol}

All tests were carried out in comfortable temperature, humidity, and light, with the subjects supine and wearing headphones (keeping their eyes closed). ${ }^{18}$ The subjects avoided tapping with a finger or foot (to avoid artefactual entrainment), confirmed by continuous visual monitoring. ${ }^{19}$ After subjects had a 20 minute period of quiet rest, we continuously monitored heart rate (RR interval) from the ECG (chest leads) and non-invasive beat to beat blood

Abbreviations: HF, high frequency; LF, low frequency; TCD, transcranial Doppler 
Table 1 Participants' characteristics

\begin{tabular}{llll}
\hline & Musicians & Non-musicians & p Value \\
\hline Number & 12 & 12 & NS \\
Men/women & $5 / 7$ & $7 / 5$ & NS \\
Age (years) & $23(1)$ & $25(1)$ & NS \\
Weight $(\mathrm{kg})$ & $61.5(3.9)$ & $62.7(2.3)$ & NS \\
Height $(\mathrm{cm})$ & $170.8(2.5)$ & $169.7(2.1)$ & NS \\
BMl $\left(\mathrm{kg} / \mathrm{m}^{2}\right)$ & $20.8(0.7)$ & $21.7(0.5)$ & NS \\
\hline
\end{tabular}

Data are mean (SEM).

BMI, body mass index; NS, not significant.

pressure by applanation tonometry at the radial artery (Pilot model; Colin tonometry, San Antonio, Texas, USA). This method faithfully tracks changes in invasive blood pressure. ${ }^{20}$ Mid-cerebral artery blood flow velocity was monitored by a $2 \mathrm{MHz}$ transcranial Doppler (TCD) probe at a depth of 35$55 \mathrm{~mm}$ through the temporal window of the non-dominant side (DWL, Sipplingen, Germany). Respiratory movements were continuously evaluated by inductive plethysmography expressed as a percentage of baseline values. In a steady state the amplitude of this signal has excellent intrasubject correlation with tidal volume recorded with a facial mask or a mouthpiece and a pneumotachograph, but avoids the respiratory modifications induced by such devices. ${ }^{12}$ End tidal carbon dioxide was monitored by a nasal cannula and side stream capnography (COSMOplus, Novametrix, Wallingford, Connecticut, USA).

Baseline recordings were taken for five minutes. Then, in random order with no intervening pauses, presentations of two minute periods of (1) slow classical, (2) fast classical, (3) dodecaphonic, (4) techno, (5) rap, and (6) raga music began. Table 2 shows details of these music tracks.

Then the tracks were repeated in a different random order but this time for four minutes. In addition, we recorded two minutes of silence, randomly inserted into either the short or long music sequences. Lastly we asked the subjects to rate their preferences for the six music tracks.

To obtain a suitably long period of music with a stable character and tempo, we chose periods with a steady orchestral or rhythmic line. Then we appended several identical sequences together to maintain a two or four minute melodic and harmonic continuity.

\section{Data acquisition and analysis}

All signals were continuously acquired on a personal computer (Apple Macintosh G3, Cupertino, California, USA) at a frequency of 600 samples/channel. Optical disk storage allowed further analysis. Breathing rate, relative tidal volume, minute ventilation, and end tidal carbon dioxide were calculated for each breath. Mean values for heart period (RR interval) and systolic blood pressure were obtained, after discarding the first 30 seconds. Baroreflex sensitivity was calculated from the same sequences of RR interval and systolic blood pressure by autoregressive power spectral analysis of the RR interval and systolic blood pressures $(\alpha$ index). ${ }^{21}$ This method is particularly well suited for relatively short term sequences (two or four minute).

We evaluated sympathovagal balance by the ratio between low (LF) (from 0.03 to $0.15 \mathrm{~Hz}$ ) and high frequency (HF) components $(0.15$ to $0.40 \mathrm{~Hz})$ of heart rate variability. ${ }^{21}$ This ratio reflects the sympathovagal balance only when the respiratory oscillations remain in the HF band. ${ }^{22}$ To remove any spurious LF due to slow respiration, we calculated the LF and HF power of the respiratory signal and then removed from the RR LF power the proportion of the LF due to respiration. Therefore, the LF:HF ratio was obtained by using LF oscillations not directly influenced by respiration.

\section{Statistical analysis}

Data are presented as mean (SEM). Analysis of variance for mixed design/repeated measures on two factors tested the effects of different music types, of duration/repetition, for the two groups. ${ }^{23}$ By looking at different aspects, or by recombining the data, we were able to obtain several types of information. We also evaluated the relation between each variable and the tempi of the tracks by linear regression analysis.

\section{Effect of music style}

An exceedingly high number of potential factors can affect the cardiovascular response to music perception, so we chose to test the contribution of various factors by comparing different music styles (table 2): harmonic, melodic, or rhythmic structure (non-syncopated versus syncopated) and tempo (rhythm speed). We graded the tracks according to each of these aspects to determine which characteristics were important for the cardiovascular and respiratory responses. Randomisation of order of presentation allowed independent unbiased assessment of these multiple factors.

\section{Effect of duration of the music and of repetition}

As a test of reproducibility and habituation, we compared the initial two minute sequence with the longer four minute sequence, as well as the initial versus the final two minutes of four minute tracks.

Non-specific effect of exposure to music

To identify any non-specific "order" effect we grouped all data in terms of order of presentation (that is, regardless of

Table 2 Characteristics of music tracks

\begin{tabular}{|c|c|c|c|c|c|}
\hline Style & $\begin{array}{l}\text { Tempo } \\
\text { (beats/min)* }\end{array}$ & $\begin{array}{l}\text { Harmonic } \\
\text { structure }\end{array}$ & $\begin{array}{l}\text { Melodic } \\
\text { structure }\end{array}$ & $\begin{array}{l}\text { Rhythmic } \\
\text { structure }\end{array}$ & Source \\
\hline Raga & 55 & Modal & Modal & Minimal & $\begin{array}{l}\text { Debabrata Chaudhuri: introduction from "Raga Maru Behag" } \\
\text { from Sitar music meditations, Music for Pleasure Ltd, Feltham, } \\
\text { UK, } 1967\end{array}$ \\
\hline Classical slow & 70 & Conventionalt & Conventional & Present & $\begin{array}{l}\text { Ludwig van Beethoven: "Adagio molto e cantabile" from the } \\
\text { Ninth Symphony Op 125, } 1823\end{array}$ \\
\hline Dodecaphonic & 76 & Absent & Absent & Absent & $\begin{array}{l}\text { Anton Webern: "Zart bewegt", from Pieces for orchestra Op } 6 \\
1909\end{array}$ \\
\hline Rap & 103 & Semimodal & Rudimentary & Strong syncopated & $\begin{array}{l}\text { Red Hot Chili Peppers: "The power of equality" from Blood } \\
\text { Sugar Sex Magik Warner Bros, } 1991\end{array}$ \\
\hline Techno & 136 & Conventional & Rudimentary & $\begin{array}{l}\text { Strong, obsessive } \\
\text { non-syncopated }\end{array}$ & $\begin{array}{l}\text { Gigi D'agostino: "You spin me round" from Tecno Fes, vol } 2 \text {, } \\
\text { Noisemaker, } 2000\end{array}$ \\
\hline Classical fast & 150 & Conventional & Conventional & $\begin{array}{l}\text { Strong non- } \\
\text { syncopated }\end{array}$ & $\begin{array}{l}\text { Antonio Vivaldi: "Presto" from "Estate", Concerto for Violin, } \\
\text { Orchestra, and Continuo no 2, Op } 8,1725\end{array}$ \\
\hline
\end{tabular}

${ }^{*}$ Tempo is measured in beats $/ \mathrm{min}$, where 1 beat $=1$ crotchet (quarter note); $†$ conventional relates to the musical culture of white subjects. 

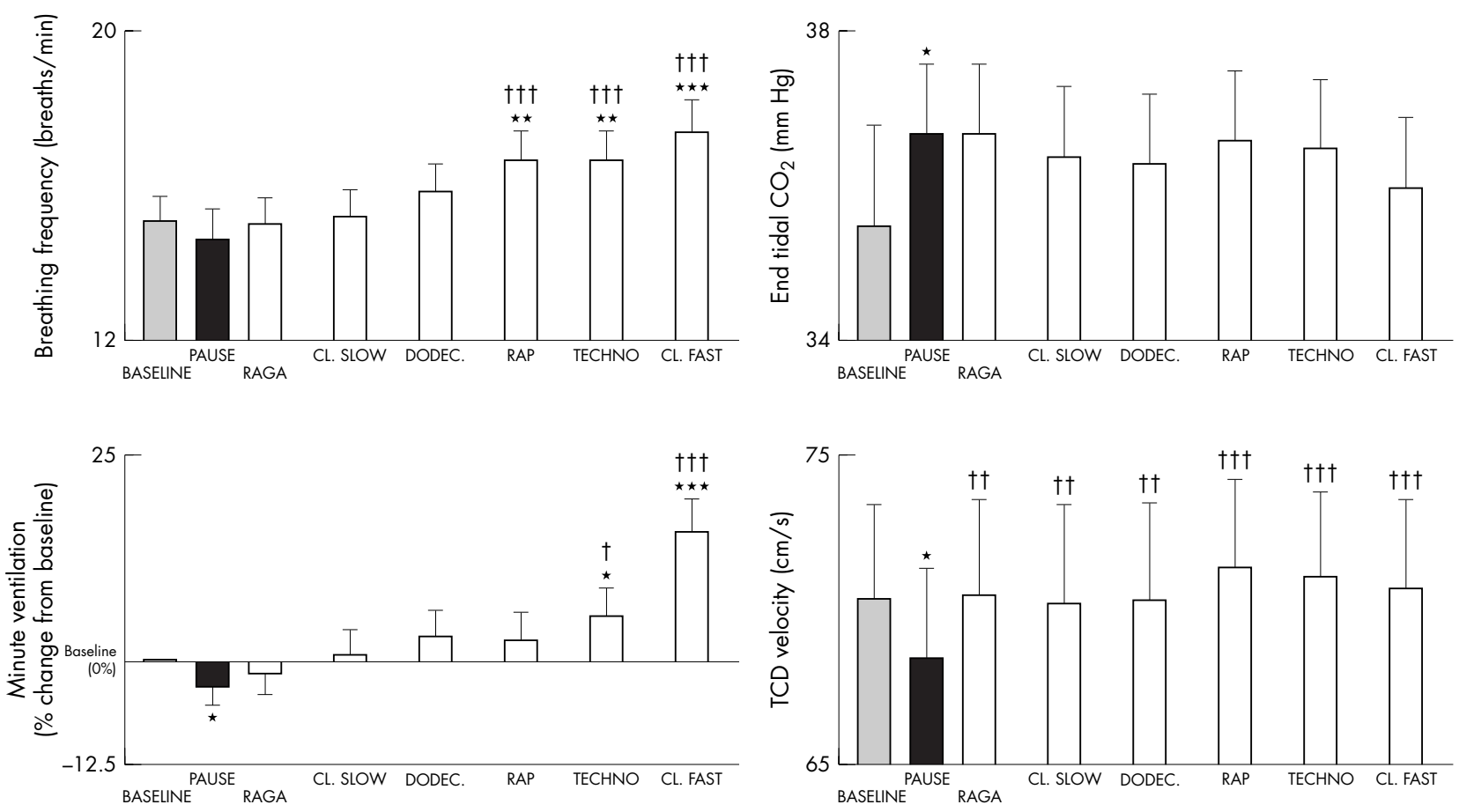

Figure 1 Responses in breathing frequency, end tidal $\mathrm{CO}_{2}$, minute ventilation, and transcranial mid-cerebral Doppler (TCD) flow velocity for each intervention. $\mathrm{CL}$, classical; DODEC, dodecaphonic. ${ }^{*} p<0.05,{ }^{* *} p<0.01$, ${ }^{* * *} p<0.001$ versus baseline; $\uparrow p<0.05$, t†p $<0.01$, t†tp $<0.001$ versus pause.

the type of music presented) — that is, we grouped all data by the first track, the second track, and so on.

\section{Effect of music training}

We compared musicians versus non-musicians.
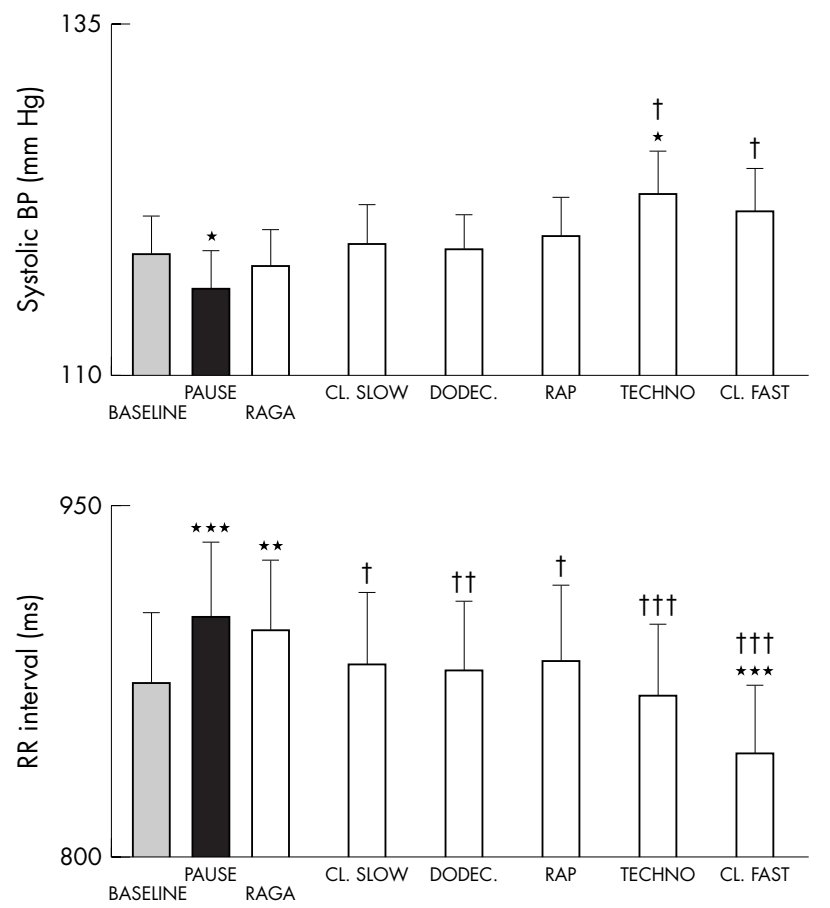

\section{RESULTS}

Effect of music styles

Figures 1 and 2 show the effects of different music styles. Faster tempi induced significant increases in ventilation, breathing rate, TCD, systolic and diastolic blood pressures,
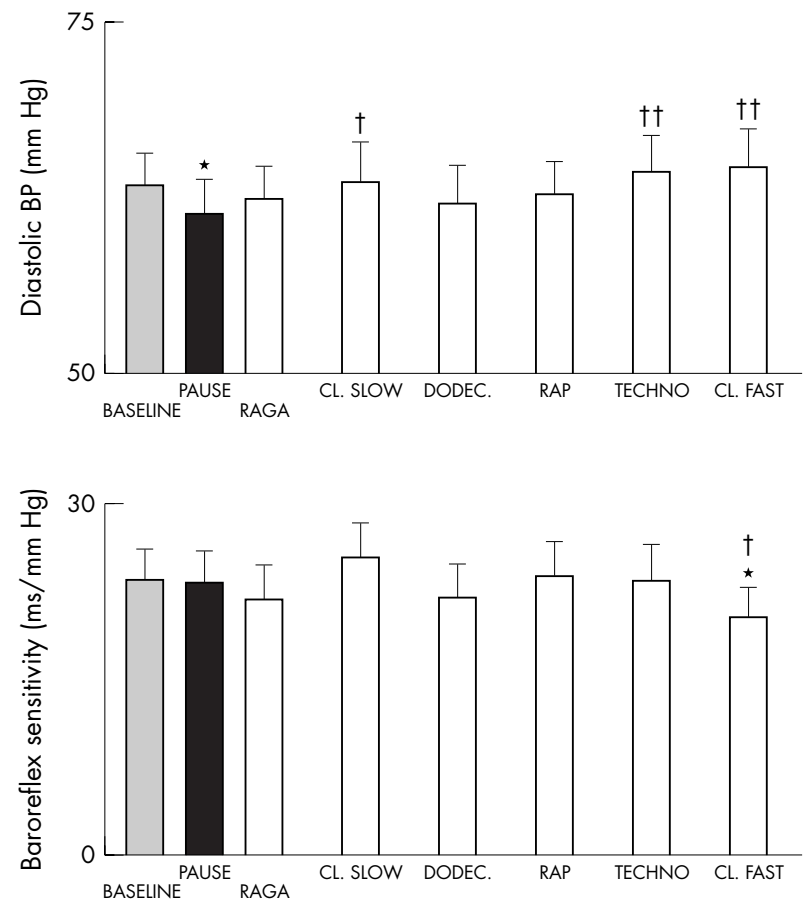

Figure 2 Responses in blood pressure (BP), interbeat period (RR interval), and baroreflex sensitivity for each intervention. The tracks are arranged in order of increased tempo. ${ }^{*} p<0.05,{ }^{* *} p<0.01,{ }^{* * *} p<0.001$ versus baseline; $\uparrow p<0.01,+\uparrow p<0.01,+\dagger \dagger p<0.001$ versus pause. 


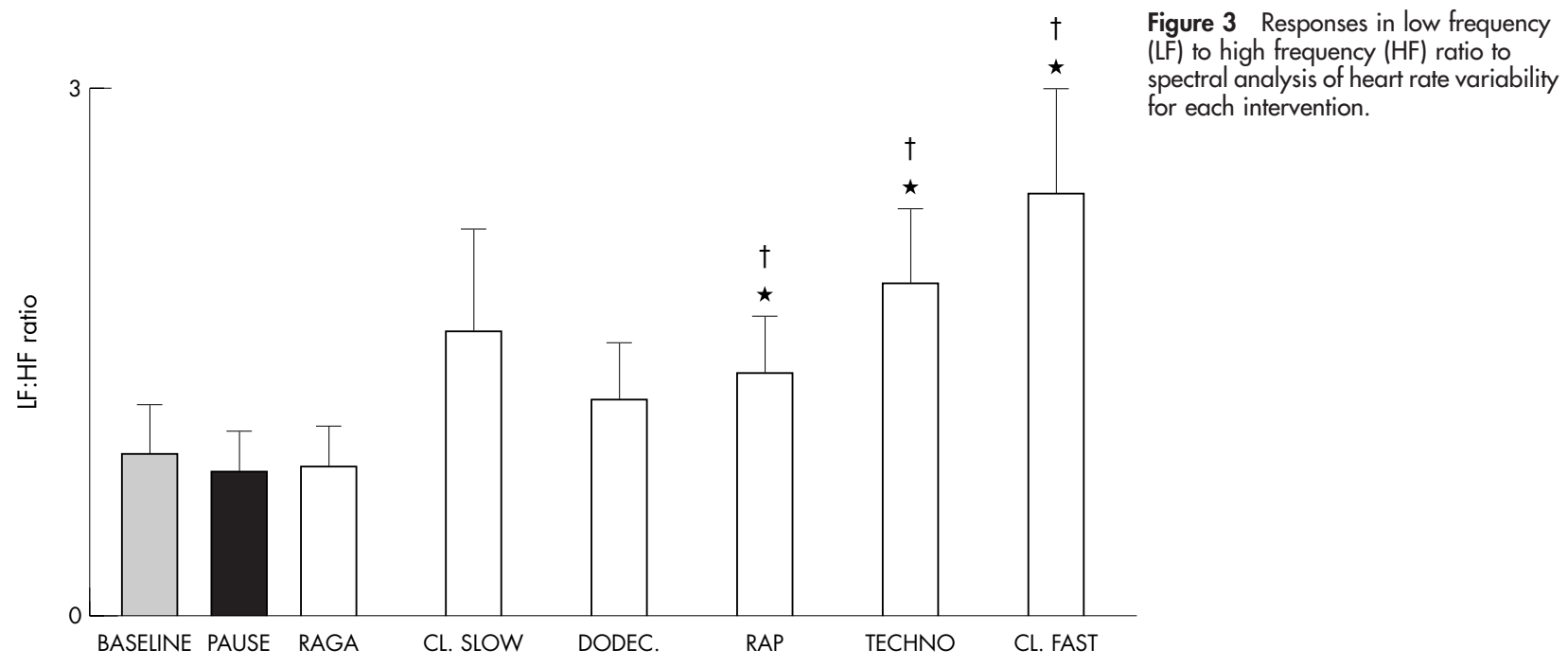

and heart rate (RR interval), tended to reduce baroreflex sensitivity, and induced a progressive increase in the LF:HF ratio (fig 3). No significant changes were seen in end tidal carbon dioxide. In contrast, slower music had a proportionally smaller effect and raga induced a significantly larger fall in heart rate (increase in RR interval, $p<0.01$ ), even compared with baseline or any other music tracks.

These effects appeared to depend on the speed of the music rather than on the style. For example, classical and techno styles induced similar results when similarly fast; raga, classical, and dodecaphonic music, all similarly slow, reduced cardiorespiratory responses.

The breathing rate (fig 4), and the LF:HF ratio (fig 5) increased significantly with increasing tempo. No other aspects of the six music tracks appeared to be relevant. There ratio of tempo to breathing rate was 4.1 (0.3) for raga, $4.7(0.4)$ for classical slow, $5.2(0.3)$ for dodecaphonic, 6.53 (0.4) for rap, 8.0 (04) for techno, and 8.7 (0.6) for classical fast. These results were not influenced by the music preferences of the subjects. Table 3 shows that their preferences for structured harmonic and melodic music were independent of the tempo.

\section{Effect of repetition of music and reproducibility}

Later repetition caused no significant overall difference (analysis of variance) in response during any individual track, with the notable exception of TCD, which was notably and significantly $(p<0.001)$ lower on the second presentation, even below baseline. Except for TCD, repetition of the music induced no other habituation even when listened to for a longer period (four versus two minutes), and the same music style (in short versus longer tracks) induced nearly identical effects. Lastly, the effects of the first two minutes of a four minute track were not significantly different from the effects of the final two minutes or of the original two minute version (except for TCD; see above).

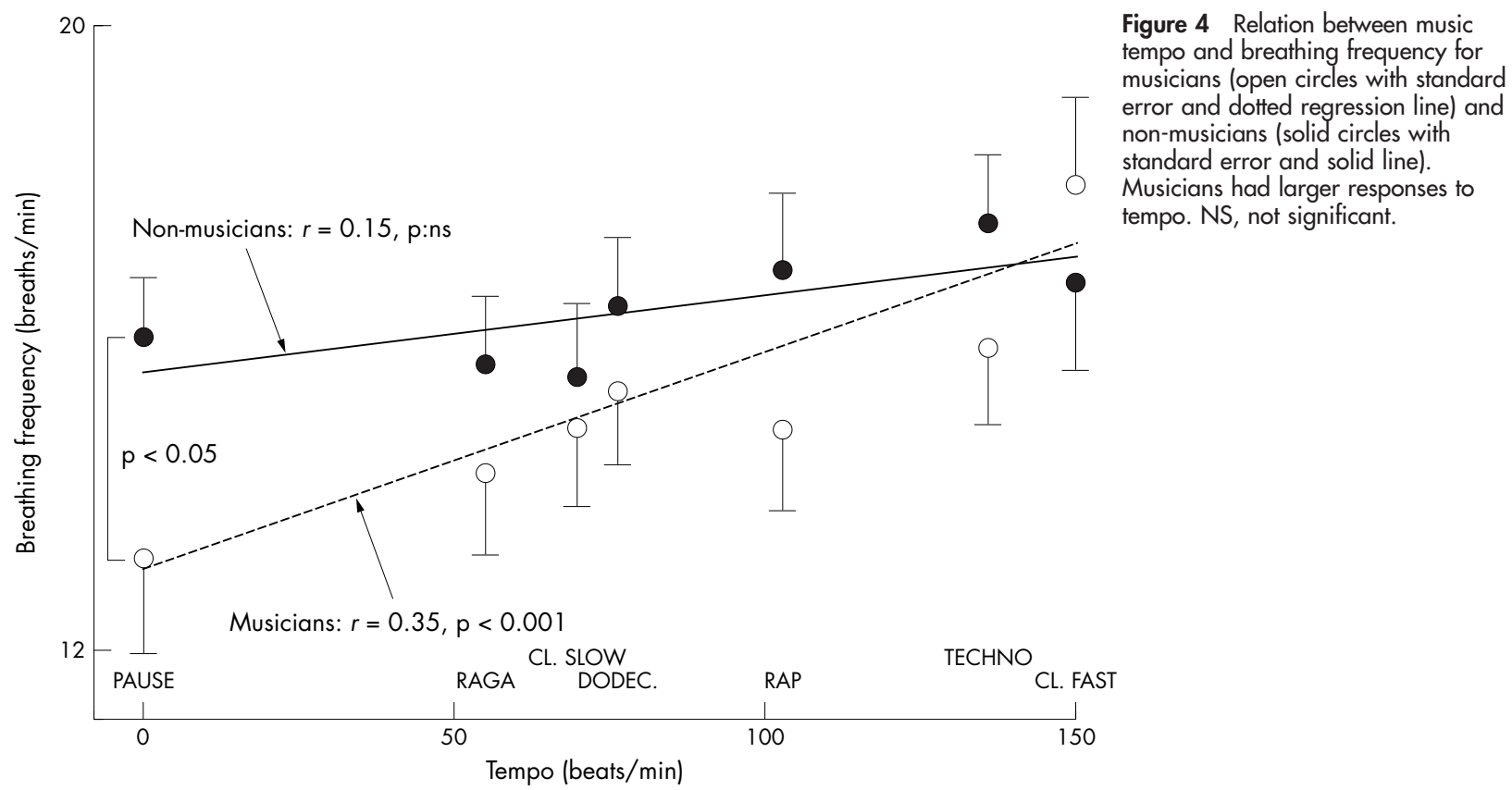

Figure 4 Relation between music empo and breathing frequency for usicians lopen circles with standard non-musicians (solid circles with standard error and solid line). Musicians had larger responses to 


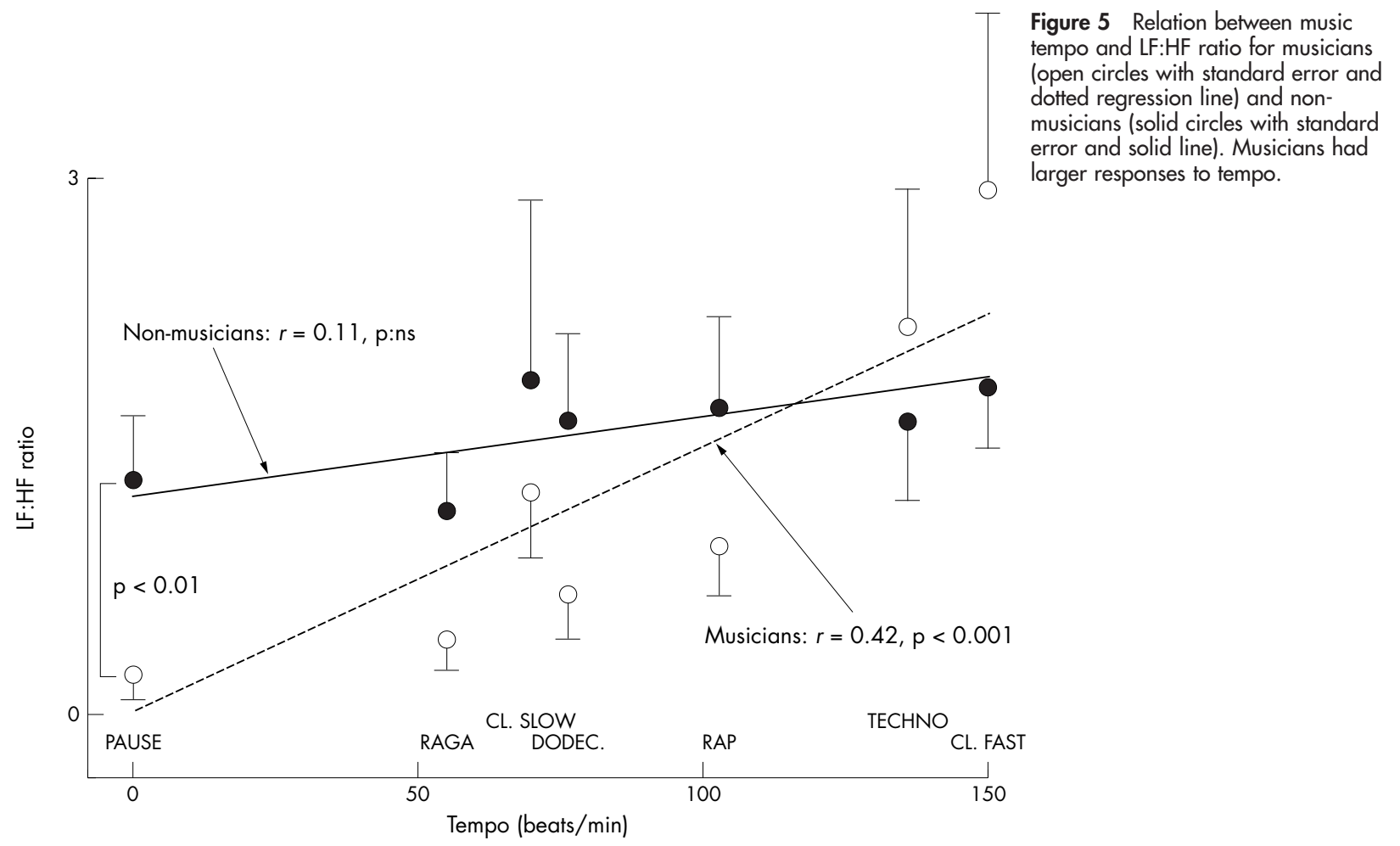

Non-specific effect of exposure to music (order effect) When we recombined the entire dataset by order of presentation (first music track, second music track, and so on) we observed (fig 6) a significant order effect, independent of music style. This overall effect was highly significant for TCD, which increased slightly at the beginning of the experiment and then progressively dropped below baseline. Breathing rate and ventilation also increased initially at the beginning of the experiment, with subsequent inconsistent changes. Systolic and diastolic pressures and heart rate trends were similar to that of TCD; these did not reach significance. No identifiable trends were seen in end tidal carbon dioxide, baroreflex sensitivity, or LF:HF ratio.

\section{Effect of a randomly inserted pause between music} tracks

In contrast, the two minute rest randomly introduced into the sequence of tracks was characterised by the lowest systolic and diastolic blood pressure, heart rate (longest RR interval), and minute ventilation and (in musicians) LF:HF ratio and by the highest end tidal carbon dioxide (figs 1-5).

Table 3 Musical preferences

\begin{tabular}{|c|c|c|c|c|c|}
\hline & \multicolumn{2}{|l|}{ Musicians } & \multicolumn{2}{|c|}{ Non-musicians } & \multirow[b]{2}{*}{$p$ Value } \\
\hline & Score & $\begin{array}{l}\text { Order of } \\
\text { preference }\end{array}$ & Score & $\begin{array}{l}\text { Order of } \\
\text { preference }\end{array}$ & \\
\hline Classical fast & $1.5(0.2)$ & 1 & $1.3(0.2)$ & 1 & NS \\
\hline Classical slow & $1.7(0.2)$ & 2 & $2.1(0.3)$ & 2 & NS \\
\hline Rap & $3.3(0.3)$ & 3 & $3.7(0.3)$ & 3 & NS \\
\hline Raga & $4.3(0.3)$ & 4 & $4.0(0.3)$ & 4 & NS \\
\hline Techno & $4.6(0.3)$ & 5 & $4.6(0.5)$ & 5 & NS \\
\hline Dodecaphonic & $5.4(0.2)$ & 6 & $5.2(0.3)$ & 6 & NS \\
\hline
\end{tabular}

\section{Effect of music training}

There were no major differences between musicians and nonmusicians but the analysis of variance model showed both at baseline and during listening that musicians had a significantly lower respiratory rate $(p<0.05)$ and increased their breathing rate more than non-musicians in response to increasing tempi (fig 4). During the pause, they had a lower respiratory rate and lower LF:HF ratios than non-musicians (figs 4 and 5). Table 3 shows that the order of music style preferences was identical in the two groups of subjects. No other aspects of the music tracks induced differences in the two groups.

\section{DISCUSSION \\ Main findings}

This study has discovered several novel features concerning the effects of music on the autonomic control of breathing and circulation, which may have wider implications beyond cardiorespiratory regulation.

An important order effect was seen in both musicians and non-musicians, which, to our knowledge, has been previously neglected.

Passive listening to music accelerates breathing rate and increases blood pressure, heart rate, and the LF:HF ratio (thus suggesting sympathetic activation) proportional to the tempo and perhaps to the complexity of the rhythm. The music style or a person's music preference seems less important.

Musicians breathed faster with faster tempi and had slower baseline breathing rates than non-musicians.

A randomly inserted short pause (two minutes) decreased blood pressure, minute ventilation, heart rate, and the LF:HF ratio in trained subjects. This relaxation effect was even greater than that seen at the end of five minutes of quiet relaxation at baseline. There was no evidence of autonomic habituation to any given music, except for cerebral blood flow, which progressively fell with time, regardless of the 

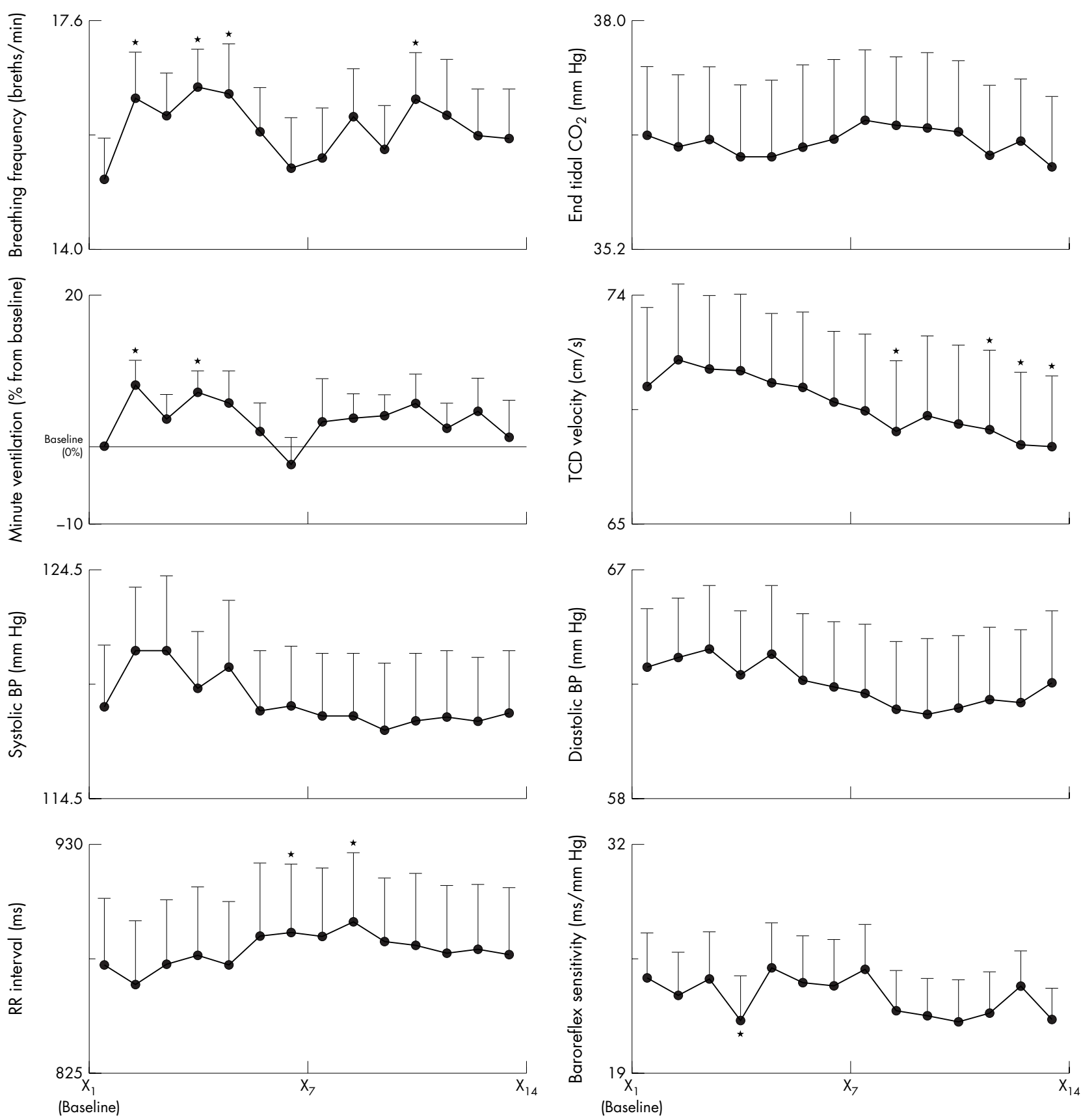

Figure 6 Responses plotted by presentation order rather than music style. X1 is baseline and X2 to X14 are the serial responses to six music tracks played twice plus one pause, all presented in random order. Note progressive trends related to order of presentation. ${ }^{*} p<0.05$.

order of music presentation. These effects were consistent during longer and shorter presentations of the same music.

At variance with the rest of the data, the TCD was significantly reduced with progression of exposure to music (order effect). Under resting conditions cerebral blood flow is mainly responsive to the local metabolic demand. Thus, during long continuous exposure to music (fig 6) and particularly during a pause (fig 1), a major drop in TCD velocity may reflect reduced metabolic demand, which is not in contrast with previous findings of a selective increase in cerebral blood flow in specific areas during listening to music. ${ }^{24}$ A previous study (during a short term comparison) reported a slight increase in response to music in the right hemisphere, which is essentially confirmed in our study, particularly during more rhythmic tracks (fig l) and in comparison with the pause. ${ }^{13}$ Owing to technical limitation, we could not assess hemispheric differences in our study. However, such differences were small in quantitative terms. ${ }^{13}$

Whether the effects observed in our study were secondary to respiratory entrainment or to a direct sympathetic stimulation by arousal remains speculative..$^{25}$ The ratio of tempo to respiratory rate was close to the music structure in the slowest (in raga and classical slow, about one breath for four crotchets) and fastest tracks (in techno and classical fast, one breath for eight crotchets), suggesting respiratory entrainment, but this was clearly absent in the intermediate rhythms. All variables were related to the tempo but not to music preference. This suggests that perhaps both respiratory entrainment by music and direct arousal were coexistent and interrelated-in fact, the increase in breathing rate in itself 
might have contributed to the increase in sympathetic activity. ${ }^{26}$

Thus, overall, we observed an arousal effect proportional to the speed of the music, with slower rhythms inducing relaxation. Even greater relaxation was induced by a randomly inserted two minute pause, suggesting that a pause (or perhaps a slowing of music tempo) may be crucially important in determining some of the relaxing effects of music.

One can speculate whether specific types of music are potentially useful as a clinical tool to modulate breathing and sympathetic arousal. Classical Greek philosophers thought that music and sports were two fundamental aspects of health. ${ }^{27}$ Newer studies emphasise the value of music in lowering stress. ${ }^{2-4}$ Meditative or slow classical music can lower neurohumoral markers of stress and thrombotic activity at rest, in contrast to the increase found with physical exercise. ${ }^{1} 1428$

How does music resemble other relaxation techniques Previous studies suggested that meditative music decreased heart rate, blood pressure, and plasma catecholamines. ${ }^{1}$ Our study confirmed these findings only partially. Slow meditative music increased the RR interval but had no significant effect on respiration and blood pressure. Instead, our data showed a consistent increase in all these variables (from baseline or from the pause) with increasing speed of music. To maintain a stable style of music we (and most previous researchers) selected periods of music with a relatively constant speed and rhythm (see methods). However, composers (even of techno music) always include pauses, even in the fastest rhythms, and in general alternate fast and slow tempi. Interestingly, it was precisely during this short intermission, rather than during music, that we observed the greatest evidence of relaxation, as compared with baseline or music tracks.

One speculative possibility arising from this effect is that music, and particularly fast tempi, induce an arousal resulting from focused attention, similar to the effect of reading silently. ${ }^{22} 2529$ This may be induced or amplified by respiratory entrainment. During a pause, or a slower tempo, arousal (and perhaps attention) is released, leaving the subject in a state of relaxation. In a typical relaxation technique the subject is encouraged firstly to concentrate attention on a specific physical or mental object (for example, during music in the present case). ${ }^{30}$ Then, the subject is encouraged to release attention (for example, during pause). This final condition is important in achieving a relaxed state. Thus, music may achieve the same effect by alternating faster and slower rhythms or pauses, or simply after its cessation. Other studies have proved that, at any given level of physical exercise, music (particularly at faster tempi) can lower the heart rate and the level of perceived exertion by a similar distracting effect. ${ }^{4-6}$

\section{Conclusions}

Even short exposure to music can induce measurable and reproducible cardiovascular and respiratory effects, leading to a condition of arousal or focused attention that is proportional to the speed of the music and that may be induced or amplified by respiratory entrainment by the music's rhythm and speed. This effect is independent (at least under the experimental conditions of our study) of a person's preference or of repetition and habituation, and is clearer when the rhythmic structure is simpler. This was perhaps less evident in non-rhythmic or syncopated music. A pause in the music induces a condition of relaxation greater than that preceding the exposure to music and leads to the speculation that music may give pleasure (and perhaps a health benefit) as a result of this controlled alternation between arousal and relaxation. It may be viewed as an alternative technique of relaxation or meditation, without involving the active participation of the subject. Previous training in music enhances the subject's ability to respond to music rhythm, since musicians learn to synchronise breathing with the music phrase. In conclusion, the present study indicates that appropriate selection of music, by alternating fast and slower rhythms and pauses, can be used to induce relaxation and reduce sympathetic activity and thus may be potentially useful in the management of cardiovascular disease.

\section{ACKNOWLEDGEMENTS}

We gratefully acknowledge the technical assistance of Nicolò Bernardi, Gaia Casucci, MD, Stefano Castoldi, Marianna Boso, MD, Francesca Cantoni, and all the subjects who volunteered for the study.

\section{Authors' affiliations}

L Bernardi, C Porta, Department of Internal Medicine, IRCCS S Matteo, University of Pavia, Pavia, Italy

P Sleight, Department of Cardiovascular Medicine, John Raddliffe Hospital, Oxford, UK

Competing interests: none declared.

\section{REFERENCES}

1 Mockel M, Rocker L, Stork T, et al. Immediate physiological responses of healthy volunteers to different types of music: cardiovascular, hormonal and mental changes. Eur J Appl Physiol Occup Physiol 1994;68:451-9.

2 White JM. Effects of relaxing music on cardiac autonomic balance and anxiety after acute myocardial infarction. Am J Crit Care 1999;8:220-30.

3 Lee $\mathbf{D}$, Henderson A, Shum D. The effect of music on preprocedure anxiety in Hong Kong Chinese day patients. J Clin Nurs 2004;13:297-303.

4 Szmedra L, Bacharach DW. Effect of music on perceived exertion, plasma lactate, norepinephrine and cardiovascular hemodynamics during treadmil running. Int J Sports Med 1998;19:32-7.

5 Thornby MA, Haas F, Axen K. Effect of distractive auditory stimuli on exercise tolerance in patients with COPD. Chest 1995;107:1213-7.

6 Szabo A, Small A, Leigh M. The effects of slow- and fast-rhythm classical music on progressive cycling to voluntary physical exhaustion. J Sports Med Phys Fitness 1999;39:220-5.

7 Thaut MH, Kenyon GP, Schaver ML, et al. The connection between rhythmicity and brain function: implications for therapy of movement disorders. IEEE Eng Med Biol Mag 1999; 18:101-8.

8 Schaver M, Mauritz KH. Musical motor feedback (MMF) in walking hemiparetic stroke patients: randomized trials of gait improvement. Clin Rehabil 200, 17:713-22.

9 Pacchetti C, Aglieri R, Mancini F, et al. Active music therapy and Parkinson's disease: methods. Funct Neurol 1998;13:57-67.

10 Sambraus HH, Hecker PA. [Effect of sound on milk production in cows]. Berl Munch Tierarztl Wochenschr 1985;98:298-302.

11 Orem J, Trotter RH. Behavioral control of breathing. News Physiol Sci 1994;9:228-32.

12 Shea SA. Behavioural and arousal-related influences on breathing in humans. Exp Physiol 1996;81:1-26.

13 Evers S, Dannert J, Rodding D, et al. The cerebral haemodynamics of music perception: a transcranial Doppler sonography study. Brain $1999 ; 122: 75-85$.

14 Gerra G, Zaimovic A, Franchini D, et al. Neuroendocrine responses of healthy volunteers to 'techno-music': relationships with personality traits and emotional state. Int J Psychophysiol 1998;28:99-111.

15 Krumhansl CK. AN exploratory study of musical emotions and psychophysiology. Can J Exp Psychol 1997;51:336-52.

16 Bernardi $L$, Sleight $P$, Bandinelli $G$, et al. Effect of rosary prayer and yoga mantras on autonomic cardiovascular rhythms: comparative study. BMJ 2001;323:1446-9.

17 Cysarz D, von Bonin D, Lackner $\mathrm{H}$, et al. Oscillations of heart rate and respiration synchronize during poetry recitation. Am J Physiol Heart Circ Physiol 2004;287:H579-87.

18 Asmussen E. Regulation of respiration: "the black box". Acta Physiol Scand $1977 ; 99: 85-90$.

19 Ebert $D$, Hefter $H$, Binkofski $F$, et al. Coordination between breathing and mental grouping of pianistic finger movements. Percept Mot Skills 2002; $95: 339-53$.

20 Kemmotsu O, Ueda M, Otsuka H, et al. Arterial tonometry for noninvasive, continuous blood pressure monitoring during anesthesia. Anesthesiology 1991 ;75:333-40. 
21 Malliani A, Pagani M, Lombardi F, et al. Cardiovascular neural regulation explored in the frequency domain. Circulation 1991;84:482-92.

22 Bernardi L, Wdowczyk-Szulc J, Valenti C, et al. Effects of controlled breathing, mental activity and mental stress with or without verbalization on heart rate variability. J Am Coll Cardiol 2000;35:1462-9.

23 Bruning JL, Kintz BL. Computational handbook of statistics. Glenview: Scott Foresman, 1968:72-83

24 Nakamura S, Sadato N, Oohashi T, et al. Analysis of music-brain interaction with simultaneous measurement of regional cerebral blood flow and electroencephalogram beta rhythm in human subjects. Neurosci Lett 1999;275:222-6.

25 Haas $F$, Distenfeld S, Axen K. Effects of perceived musical rhythm on respiratory pattern. J Appl Physiol 1986;61:1185-91.
26 Francis DP, Coats AJS, Ponikowski P. Chemoreflex-baroreflex interactions in cardiovascular disease. In: Bradley DT, Floras JS, eds. Sleep apnea: implications in cardiovascular and cerebrovascular disease. New York: Dekker, 2000:33-60.

27 Plato. The Republic, book III. London: Heinemann, 1963:286-7.

28 Molz AB, Heyduck B, Lill $H$, et al. The effect of different exercise intensities on the fibrinolytic system. Eur J Appl Physiol Occup Physiol 1993;67:298-304.

29 Conway J, Boon N, Jones JV, et al. Involvement of the baroreceptor reflexes in the changes in blood pressure with sleep and mental arousal. Hypertension 1983;5:746-8.

30 Benson H, Rosner BA, Marzetta BR, et al. Decreased blood-pressure in pharmacologically treated hypertensive patients who regularly elicited the relaxation response. Lancet, 1974;i, 289-91.

\section{IMAGES IN CARDIOLOGY}

\section{Percutaneous transluminal removal of intracardiac vegetations}

doi: $10.1136 /$ hrt.2005.069880

A 59 year old man was hospitalised for clinical signs of sepsis (methicillin resistant Staphylococcus aureus) with two large bacterial vegetations adherent on his automatic implantable cardioverter-defibrillator (AICD) lead in the right atrium and superior vena cava (panel A). Eight weeks earlier, septic ulcers first appeared on both chronic ischaemic legs (obstructive peripheral arterial disease) due to a 30 year history of insulin dependent diabetes mellitus (including diabetic nephropathy with chronic nephrodialysis since five years previously). The AICD had been implanted 15 months earlier, and two cardiac aortocoronary revascularisation operations had been carried out two and nine years earlier.

Due to the remaining septic source on both ischaemic legs which showed no improvement despite antibiotic treatment, concerns over the disturbed secondary wound healing, as well as the past history of two cardiac revascularisations, the cardiac surgeon refused to extirpate the two vegetations surgically. The decision for the first percutaneous vegetectomy was made.

In order to prevent a major pulmonary embolism, an Antheor vena cava filter (MediTech, Boston Scientific/ Scimed, Inc) was inserted into the main pulmonary artery (panel B). For percutaneous extirpation of the two large vegetations, a 10 French multipurpose guiding catheter

(Cordis) was inserted from the right vena femoralis under continuous transoesophageal guidance (panel B). For manoeuvring the 10 French catheter opening directly in front of the vegetations, an 8 French Judkins right guiding catheter was used. Using a $50 \mathrm{ml}$ syringe the first vegetation was successfully evacuated through the 10 French guiding catheter. The second (larger) vegetation could only be evacuated into the tip of the guiding catheter. Through continuous vigorous suction, the vegetation could be fixed there and subsequently removed from the AICD lead by pulling retrogradely. For the final extirpation, a surgical incision of the vena femoralis was done (panel C). The infected AICD leads were successfully removed the next day. Thrombus with large amounts of fibrin and leucocytes with basophilic sediment (as a sign of bacterial invasion) were observed histologically. After the source of the sepsis was removed, the patient improved dramatically and became afebrile and aseptic during the following days. For treatment of the secondary source of infection (ischaemic leg ulcers) percutaneous Excimer laser assisted angioplasty was successfully carried out subsequently.
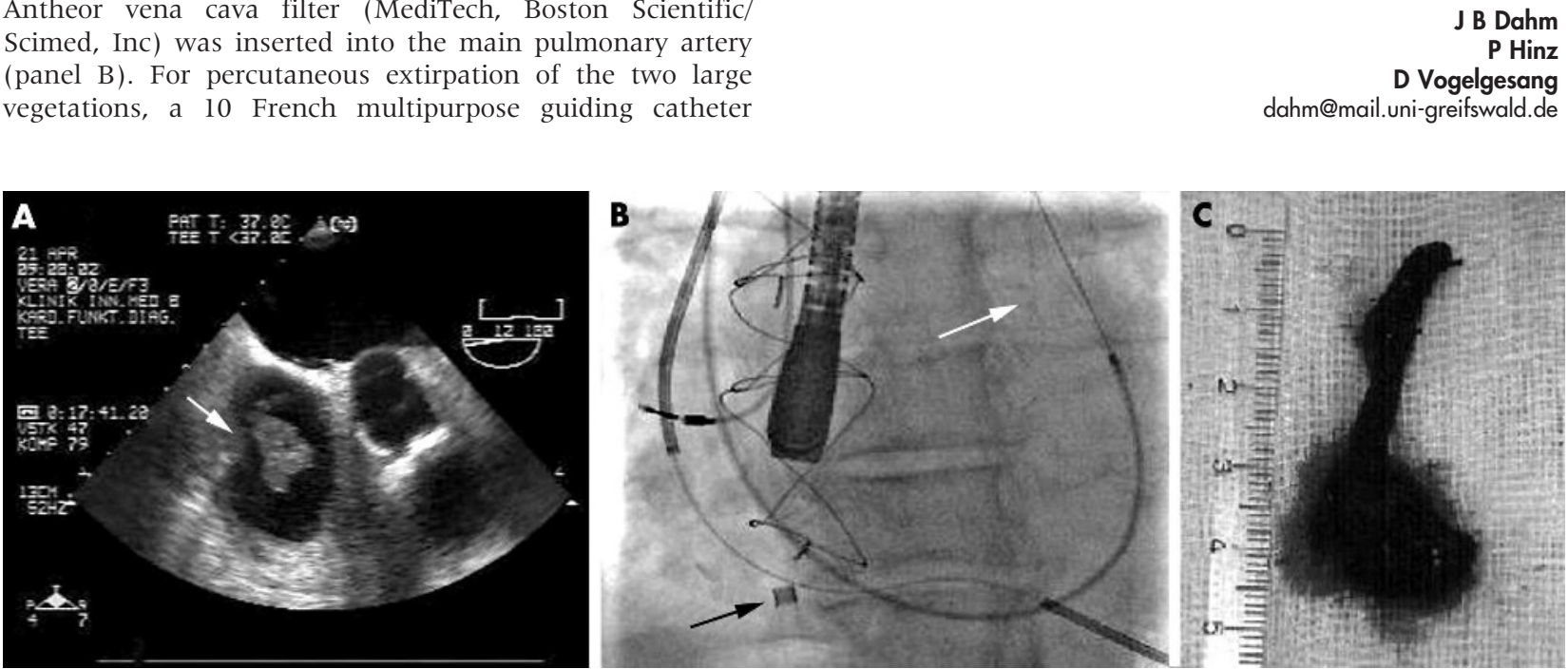

(A) Transversal transoesophageal echocardiography of one of the two vegetations adherent on the AICD lead (arrow) in the right atrium. (B) Posterior anterior $x$ ray showing the 10 French guiding catheter in the right atrium (black arrow) and the cava filter in the main pulmonary artery (white arrow). (C) Extirpated vegetation $(4.5 \times 2.0 \mathrm{~cm})$. 